\title{
Correction to: A Novel Approach for Establishing Design Criteria for Refugees' Shelters
}

\author{
Rania Aburamadan (D) and Claudia Trillo (D)
}

\section{Correction to: \\ Chapter "A Novel Approach for Establishing Design Criteria for Refugees' Shelters" in: F. Calabrò et al. (Eds.): \\ New Metropolitan Perspectives, SIST 100, https://doi.org/10.1007/978-3-319-92099-3_48}

In the original version of the book, the following belated correction has been incorporated: The author's last name has been changed from Abumaradan to Aburamadan in chapter 48 . The book and the chapter have been updated with the change. 